off the graph. Corresponding corrections were then applied to the value of $(D-R)$ for each star.

The resulting values of the differences were then arranged in order of $x$, and again means were formed and plotted for groups of 20 stars. The graph showed a very small scale correction which was then applied to the differences.

The final corrected values of the individual differences were then plotted. They show some small remaining systematic effects. It is anticipated that similar studies for additional plates will permit the isolation of such effects. The differences, as they stand, give a probable error for a setting of 0.56 micron. This value may be somewhat improved if systematic effects can be allowed for.

The measures here described indicate that the automatic engine will measure in one working day as many stars as a good observer can measure visually in a week (the observer can measure for only a few hours a day). The weight of an automatic measurement is about four times that of a visual one. Moreover, the precision of the photoelectric setting itself is still higher, the overall accuracy in the present case being limited by the engine rather than the setting. Dr. Curtis commented on the presence of errors of the order of a micron due to oil on the screw; such errors, of course, are more apparent with the new accuracy of setting. In designing a new automatic engine, one would consider the use of some other devices instead of the screw, such as an interferometer or even a scale. It should be recalled that the screw has replaced the scale to a large extent because of the additional settings and computations required with the use of a scale. The introduction of automatic measurement and computation makes it necessary to re-evaluate these factors.

\section{REFERENCES}

I. W. J. Eckert, Punched Card Methods in Scientific Computation, Ch. 9, ro, I940.

2. W. J. Eckert and R. F. Haupt, The Printing of Mathematical Tables, Math. Tables and Aids to Computation 2, $197,1947$.

3. W. J. Eckert, Electronics and Electromagnetic Measuring, Computing and Recording Devices, Harv. Obs. Monographs No. 7, p. I69, 1948.

4. Trans. Yale Astr. Obs. II, New Haven, 1939.

Watson Scientific Computing Laboratory, New York, N.Y. 1953 September.

\title{
SOME ASTROMETRIC PROBLEMS OF GALACTIC STRUCTURE
}

\section{By W. W. MORGAN}

\begin{abstract}
Attention is called to the importance of deriving proper motions of the highest accuracy for $\mathrm{O}$ stars which do not appear to be members of associations. These stars may be found to be moving with large space velocities, and the determination of the direction of these velocities may give a clue as to the place of origin.
\end{abstract}

The short time-scale for the existence of the O-type stars results in certain problems of astrometric interest. We have the expansions of some $\mathrm{O}$ associations as predicted by Ambartzumian; these have been confirmed observationally by the work of Blaauw. In addition, certain considerations indicate astrometric problems of a more general nature; these are considered with the general overall distribution of the $\mathrm{O}$ stars.

The work of Ambartzumian and his associates, followed by visual observations with the 40-inch refractor by Morgan and photographic investigations by Stewart Sharpless with the 60-inch Mount Wilson reflector, allow certain general conclusions to be drawn concerning the $\mathrm{O}$ stars; they may be summarized as follows.
The normal situation of an $\mathrm{O}$ star is, (I) as the brightest component of a visual multiple system ; (2) this multiple system is surrounded by a looser clustering; and (3) the clustering is part of a larger $\mathrm{O}$ association. The Orion association is a good example of the complex system just described; a considerable number of additional instances are now known and under investigation.

A large percentage of all known $\mathrm{O}$ stars conform to the above scheme; however, it is the isolated stars of early type which are probably of the greatest astrometric interest.

Two completely independent methods indicate that the lifetime of the $O$ stars in their present condition is of the order of $\mathrm{IO}^{6}-\mathrm{IO}^{7}$ years. This age is based on considerations of the energy 
generation and the observed expansions of the associations as determined by Blaauw.

Since there are reasons to assume that the $\mathrm{O}$ stars were created in regions similar to that in the neighborhood of the Orion nebula (high density of interstellar material), we have the problem of explaining the existence of isolated $\mathrm{O}$ stars which are situated at great distances from their probable birthplace. It seems reasonable to adopt as a hypothesis that such isolated objects form parts of rapidly expanding associations as yet unknown.

An interesting case of this sort has recently been investigated by Blaauw and Morgan; the star HD 34078 (AE Aur, O9.5 V, $m=5.8$, distance from sun $\sim 525 \mathrm{pc}$ ) has a proper motion of 0 ". 040 ; this results in a tangential velocity of I I $7 \mathrm{~km} / \mathrm{sec}$, after correction for solar motion. The radial velocity, after elimination of solar motion, is $+5 \mathrm{I} \mathrm{km} / \mathrm{sec}$. The space motion appears to be directly away from the region of the Orion nebula. With the star's space velocity of $128 \mathrm{~km} / \mathrm{sec}$, the distance from the Orion nebula would have been travelled in $2.7 \times \mathrm{IO}^{6}$ years; this is a reasonable estimate for the lifetime of such a star, and it seems quite possible that $\mathrm{AE}$ Aur actually originated in the Orion association.

Among the $\mathrm{O}$ stars which have been observed at Yerkes there are several cases of objects located in rather high galactic latitude; these would be among the most promising cases to investigate for large space motions by accurate new observations of proper motions. Five such objects are listed in Table I ; they are all at distances which would permit measurable proper motions to be observed, if their space motions are similar to that of AE Aur ; in this case proper motions of the order of o".oro might be expected.

\begin{tabular}{|c|c|c|c|c|c|c|}
\hline Hd No. & $l$ & $b$ & MK Type & $m$ & $6 E_{1}$ & $m_{0}-M$ \\
\hline I337 (AO Cas) & $85^{\circ} \cdot 6$ & -10.9 & O9 III & 6.1 & 0.36 & II. 8 \\
\hline 14633 & 108.9 & -17.4 & $08 \mathrm{~V}$ & $7 \cdot 3$ & - & Ir. 8 \\
\hline 306I4 ( $\alpha$ Cam) & III.4 & +14.9 & $\mathrm{O} 9.5 \mathrm{Ia}$ & $4 \cdot 4$ & .72 & I0.7 \\
\hline 60848 & 170.3 & +19.0 & O8 V :pe & 7.2 & .24 & II. 5 \\
\hline $9352 \mathrm{I}$ & 150.1 & +63.6 & $\mathrm{O}_{9} \mathrm{Vp}$ & 6.9 & .06 & II. I \\
\hline
\end{tabular}

The columns are self-explanatory, with the possible exception of column six, which lists the estimated total absorption from the color excess as measured by Stebbins, Huffer and Whitford.

The star $\alpha$ Cam is of very high luminosity and is in some respects the most interesting case; it does not seem to belong to any association, and a detailed investigation of its space motion would be of special interest.

Yerkes Observatory, Williams Bay, Wis., 1953 September. 